PRAXIS

ecucativa

Universidad Nacional de La Pampa

Facultad de Ciencias Humanas

Instituto de Ciencias de la Educación

para la investigación interdisciplinaria

\section{† 国埐 \\ Instituto de Ciencias de la Educación

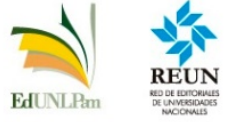

ISSN 2313-934X

SANTA ROSA, LA PAMPA, ARGENTINA

Correo electrónico: iceii@humanas.unlpam.edu.ar

Disponible en https://cerac.unlpam.edu.ar/index.php/praxis

Opciones y decisiones previas en jóvenes que migran desde el interior de la provincia de Córdoba para iniciar los estudios superiores. Artículo de Sandra María Gómez; Débora Schilling; Renata Pons y Sebastián Maldonado. Praxis educativa, Vol. 25, No 3 septiembre diciembre 2021. E - ISSN 2313-934X. pp. 1-17. https://dx.doi.org/10.19137/praxiseducativa-2021-250312

Esta obra se publica baja Licencia Creative Commons 4.0 Internacional CC BY- NC- SA Atribución, No Comercial, Compartir igual

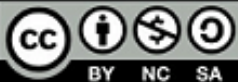

\title{
Opciones y decisiones previas en jóvenes que migran desde el interior de la provincia de Córdoba para iniciar los estudios superiores
}

Options and prior decisions in young people who migrate from the countryside of the province of Córdoba to start higher studies

Opções e decisões prévias nos jovens que migram do interior da província de Córdoba para iniciar estudos superiores

\section{Sandra María Gómez}

Universidad Nacional de Córdoba, Universidad Católica de Córdoba y Universidad Siglo 21, Argentina

sgomezvinuales@gmail.com

ORCID: 0000-0001-7322-057

\section{Débora Schilling}

Universidad Nacional de Córdoba, Argentina

schillingdebora@gmail.com

ORCID: 0000-0003-0585-1042

\section{Renata Pons}

Universidad Católica de Córdoba, Argentina

ponsrenata@gmail.com

ORCID: 0000-0001-8302-9834 


\section{Sebastián Maldonado}

Universidad Católica de Córdoba y Universidad Siglo 21, Argentina

sebamaldonado@hotmail.com

ORCID : 0000-0003-0915-6477

Recibido: 2021- 04-19 | Revisado: 2021-06-18 | Aceptado: 2021-05-31

\section{Resumen}

En el presente escrito, se comparten algunas interpretaciones de relatos de jóvenes vinculados al proceso decisorio que van viviendo respecto al hecho de tener que ir a vivir fuera del entorno familiar, movilidad justificada principalmente en una opción de continuidad de los estudios en el nivel superior, producción inscrita en una investigación denominada "Migraciones estudiantiles e inicio de carrera universitaria. Experiencias juveniles: desde el proyecto imaginado al proyecto concretado". Se trabajó desde un enfoque cualitativo longitudinal identificando aspectos de la trayectoria del y la joven, desde el sexto año del secundario hasta los dos primeros años de arribo a la ciudad de Córdoba. En esta instancia, enfocaremos en la fase preliminar al desplazamiento. Se realizaron entrevistas a jóvenes y madres, en los años 2018 y 2019, a los fines de conocer cómo se va imaginando y definiendo el proyecto de movilidad y de estudio, las opciones, expectativas y decisiones.

Palabras clave: movilidad estudiantil; universidad; estudiante; joven.

\section{Abstract}

This paper shares some interpretations of stories of young people linked to the decision-making process that they are going through regarding the fact of having to live outside the family environment, decision justified mainly in the pursuit of continuing studies at a higher level, a topic developed in a research called "Student migration and the beginning of a university career. Youth experiences: from the imagined project to the concrete project". We worked from a longitudinal qualitative approach, identifying aspects of the youngster's trajectory, from the sixth grade of secondary school to the first two years of arrival in the city of Córdoba. In this instance, we will focus on the step prior to migration. Interviews were conducted with young people and mothers, in 2018 and 2019, in order to know how the mobility and study project is imagined and defined, as well as options, expectations and decisions.

Keywords: internal migration, universities, students, young people.

\section{Resumo}

Neste escrito, são partilhadas algumas interpretações das histórias de jovens ligadas ao processo de tomada de decisão que atravessam quanto ao fato de ter que ir morar fora do meio familiar, mobilidade justificada principalmente pela opção de continuidade dos estudos no nível superior, tópico desenvolvido na pesquisa denominada "Migração de alunos e início da carreira universitária. Experiências juvenis: do projeto imaginado ao projeto concreto". Trabalhamos a partir de uma abordagem qualitativa longitudinal, identificando aspectos da trajetória do jovem, desde o sexto ano do ensino médio até os dois primeiros anos de chegada à cidade de Córdoba. Neste caso, vamos nos concentrar na fase preliminar à migração. Foram realizadas entrevistas com 
jovens e mães, em 2018 e 2019, com o objetivo de conhecer como se imaginam e se define o projeto de mobilidade e de estudo, as opções, expectativas e decisões.

Palavras-chave: mobilidade do estudante; universidade; estudante; jovem.

\section{Introducción}

En el presente escrito, se comparten algunas interpretaciones de lo que los/as jóvenes relatan en relación con el proceso que van viviendo relativo a una decisión trascendental para ellos/as, como es el hecho de tener que ir a vivir fuera del entorno familiar, espacio vincular en el que han estado durante su niñez y adolescencia. La movilidad es justificada principalmente, aunque no solamente, en un proyecto de estudio en el nivel superior.

La provincia de Córdoba cuenta con cinco universidades de gestión estatal y siete de gestión privada. La mayor parte se ubica en la capital cordobesa y algunas estatales se encuentran en el interior de la provincia. Aun con esa distribución, muchos son los/as jóvenes que deben movilizarse e instalarse en otro lugar para poder hacer estudios universitarios. Este artículo aborda aspectos del proceso anterior al desplazamiento, tiempo de decisiones en el que participan varios actores: familia, escuela y amistades. Es un hito temporal que funciona como bisagra entre la finalización de la escuela secundaria y el proyecto de inicio de estudios en la universidad. Desde el proyecto de investigación marco denominado Migraciones estudiantiles e inicio de carrera universitaria. Experiencias juveniles: desde el proyecto imaginado al proyecto concretado, se trabaja un enfoque cualitativo longitudinal en el cual se estudia la trayectoria del y la joven, desde el sexto año del secundario hasta los dos primeros años de arribo a la ciudad de Córdoba. En esta instancia de escritura, nos enfocaremos en esa fase preliminar al desplazamiento. Se realizaron entrevistas a los/as jóvenes durante el último semestre del sexto año, en los años 2018 y 2019, a los fines de conocer cómo se va construyendo el proyecto migratorio y de inicio de estudios superiores, sus opciones, expectativas y decisiones.

En nuestra investigación, una de las preguntas alude a conocer cómo se van construyendo las elecciones de carrera y de ciudad de destino para estudiar en la universidad en jóvenes del interior de la provincia de Córdoba que están en sexto año de la escuela secundaria, problema que se ha indagado de modo cualitativo a los fines de describir e interpretar las experiencias vividas por los/as jóvenes del interior de la provincia de Córdoba, durante el sexto año del nivel secundario, en relación con esas elecciones vinculadas a qué y dónde estudiar.

\section{Antecedentes}

Hay investigaciones anteriores que nutrieron y colaboraron en la problematización de nuestro tema de investigación. Habida cuenta que nos interesa aquello que algunos/as jóvenes hacen luego de sus estudios secundarios, nuestro foco está puesto en los sujetos que deciden seguir estudios superiores y que, para ello, deben migrar. 
Una investigación relevante que alimenta nuestro trabajo es la de Cleve (2016). La misma se centra en el rol que tienen los grupos familiares en las migraciones internas de los/as estudiantes, desde la localidad de Roque Pérez (provincia de Buenos Aires) hacia la ciudad de La Plata para comenzar la universidad. La pregunta de investigación se vinculó al papel que cumplen los grupos familiares en este tipo de migración estudiantil. La estrategia metodológica fue de tipo cualitativa, etnográfica, con observaciones en espacios públicos y ámbitos domésticos. La técnica, bola de nieve, fue utilizada en casi todos los casos junto al software Atlas Ti, logrando reconstruir historias de vida. Como conclusiones de su trabajo de análisis, el autor señala que cada una de las historias de los/as jóvenes entrevistados reviste su particularidad y resulta imposible pensar sus trayectorias como caminos iguales y preestablecidos. Sin embargo, es posible identificar algunas regularidades. La llegada a una ciudad diferente a la de origen, la construcción de vínculos afectivos y el ingreso a la universidad son sucesos comunes, pero experimentados de modos diferentes. La experiencia migratoria es considerada como un fenómeno que posibilita la construcción de modos de vida diferentes, de nuevas formas de sociabilidad y nuevos sentidos de responsabilidad. En dicha experiencia, no todos los/as jóvenes se encuentran en igualdad de posiciones y eso estructura trayectorias diferentes y desiguales. Las redes de contención actúan como eje central en lo que respecta a la inserción de los/as jóvenes en el nuevo contexto urbano. Señala también que la migración puede pensarse como una transición hacia un tipo particular de condición juvenil con algunas características diferentes a la de la ciudad de origen, como por ejemplo la autonomía de la familia, la autoadministración y el contacto con personas de otras localidades. En investigaciones anteriores (Gómez et al., 2016; Gómez et al, 2017; Gómez, 2019), habíamos hallado algunos aspectos semejantes a los que plantea Cleve. Ahora, nuestras preguntas giran sobre el momento inmediato anterior a la mudanza, es decir, aquellos momentos decisorios que se producen durante la última etapa de la escuela secundaria y la llegada a la ciudad de Córdoba.

Por otra parte, cabe considerar el trabajo de Mercado (2014). Dedica su estudio al análisis de las perspectivas y sentidos que construyen los/as estudiantes universitarios venidos del interior de la provincia de Córdoba, durante sus primeros años de estudio, en relación con su vida cotidiana en la ciudad capital y en la Universidad Nacional de Córdoba. Trabaja sobre tres casos de estudiantes provenientes de localidades rurales de la provincia de Córdoba, que llegaron a la universidad mediante el PECU (Proyecto de Estudiantes Campesinos en la Universidad). A partir de allí, aborda el tema de la interculturalidad, la inclusión de los/as jóvenes y los apoyos universitarios, las estrategias desplegadas por estos/as estudiantes que son la primera generación de su familia en llegar a cursar estudios de nivel superior. Se pone en tensión la idea de una educación para privilegiados según sea el origen y las condiciones objetivas de estos tres casos provenientes del interior que sostienen sus estudios ayudados por becas estudiantiles y otros soportes que colaboran en la sustentabilidad del proyecto de estudio. Nuevamente, este estudio aborda los cambios generados durante la estancia en la nueva ciudad y las particularidades de estos/as estudiantes. 
Otro antecedente recuperado ha sido la tesis de Pucci (2018) denominada Factores que inciden en el proceso de toma de decisión vocacional en jóvenes del interior que migran para continuar estudios superiores. El objetivo principal ha sido analizar los factores fundamentales que inciden en la toma de decisión vocacional en los/as jóvenes del interior que migran a otra ciudad, para continuar estudios superiores. El estudio fue de revisión bibliográfica. Sostiene el autor que los/as adolescentes, cuando cursan los últimos años del secundario, deben enfrentarse a una expectativa social que les exige dar respuestas y tomar decisiones. Aquí, conocen sus posibilidades y limitaciones y surge una conciencia de responsabilidad en relación con su futuro. Destaca que, en esta etapa, surgen las preguntas como: ¿quién soy?, ¿quién quiero ser? y ¿cómo puedo llegar a ser eso que quiero ser? El autor retoma a Erikson (1968) y recupera el planteo de la crisis de identidad al finalizar la adolescencia, acontecimiento normal que puede manifestarse en la llamada crisis vocacional por la dificultad para encontrar la genuina vocación, dificultad para elegir carrera, profesión u ocupación.

Pucci (2018) menciona que, asimismo, influyen otros factores personales, socioeconómicos, socioculturales y familiares. En el caso de los/as jóvenes del interior, se agrega otro factor que es la instancia con las universidades, siendo este un condicionante que se interrelaciona con los demás factores intervinientes en el proceso.

En nuestra investigación, siguiendo lo trabajado por Pucci, puede decirse que coinciden tres circunstancias en un mismo momento cronológico: estar transitando la etapa de la adolescencia, el pasaje del colegio secundario a los estudios superiores con su consiguiente toma de decisión vocacional dentro del contexto cultural posmoderno y, por último, el proceso migratorio. Por lo tanto, a la complejidad que caracteriza a la toma de decisión vocacional, se adicionan otras circunstancias que complejizan aún más el proceso en el caso de los/as jóvenes del interior, dado que la elección no es solo de ocupación, carrera y universidad, sino que además se adicionan otras tomas de decisiones tales como: migrar o no, la elección de una ciudad, la opción de una vivienda, la manutención, la autonomía, entre otros.

En esta misma línea, Rascovan (2015) expresa que "concluir la escuela secundaria es algo más que el fin de una etapa. Implica un proceso de cambio que requiere adaptación, reacomodamiento personal y familiar" (p. 25). Sin duda, es un momento de decisiones que afecta al y la joven y a su familia. Comienza un camino marcado por un pasaje, una transición hacia otra cosa. En la investigación, hacemos foco en el pasaje hacia los estudios superiores. Estos pasajes conllevan desprendimientos, cambios, pérdidas y conquistas. Nuestro trabajo versa sobre inquietudes que giran en torno a qué carreras eligen, qué universidades, con qué expectativas, con qué temores, qué construcciones vocacionales tienen, cómo se han generado esas inclinaciones y propensiones según las pertenencias sociales, cómo se va dando ese proceso decisorio. 


\section{Sobre el proceso decisorio: opciones, previsiones y valoraciones. Algunas precisiones teóricas}

Es necesario aludir a dos procesos que, de algún modo, convergen al momento de ir tomando decisiones en el transcurso de la última etapa de sexto año de la escuela secundaria. Es decir, aquellas definiciones que van configurando el proyecto como resultado de un proceso de elaboración y como necesidad decisoria dada la inminente terminalidad de la escuela obligatoria.

El tránsito por la escuela primaria y secundaria es un trayecto obligatorio, al que los sujetos asisten según un recorrido claramente anticipado y diseñado al cual ellos adhieren. La continuidad formativa, más allá de la educación secundaria, impone una serie de decisiones que interpelan a los/as jóvenes y los movilizan subjetivamente. Tal vez la elección del ciclo orientado, cuando tuvieron opciones, ya les supuso algún tipo de reflexión y decisión sobre cuál elegir. Una vez terminada la escolaridad obligatoria, las posibilidades a futuro se multiplican.

La escolarización es considerada como uno de los tantos lugares propicios para el proceso de socialización y formación de jóvenes y niños/as. Como señala Camarena (2000), mientras los/as jóvenes permanecen en el sistema educativo son "estudiantes", lo cual supone un lugar social claramente instituido y positivamente valorado, que involucra un conjunto de ritos que tienen valor en sí mismos -independientemente del valor propio de los aprendizajes-al implicar una cierta organización de la vida cotidiana.

Luego de finalizar la escuela secundaria, adviene otro momento que interpela a los/as jóvenes en lo vocacional. Rascovan, (2014) nos habla de caminos de la vida que se van construyendo en relación con coordenadas socio-históricas y geográficas. Esos caminos se van configurando en un recorrido que no se puede leer de modo lineal, y en el que participan múltiples actores. Las elecciones se vinculan a un hacer como estudiar, trabajar u otras actividades alrededor de las cuales se organiza gran parte de la vida cotidiana de los sujetos. Aun cuando pueda haber vaivenes en este proceso, la gran parte de los/as jóvenes entrevistados podrían corresponder a aquellos en los cuales la moratoria psicosocial ha sido posible de ser sostenida, en el sentido de las condiciones objetivas de las familias que pueden habilitar la posibilidad de que solo se dediquen por unos años a realizar la carrera. Esta lectura no implica una lectura inflexible de itinerarios prefijados, pero, en estos grupos, la futura trayectoria puede verse de modo menos incierto que para otros grupos de jóvenes.

En nuestra investigación, trabajamos con jóvenes que están pensando que, luego de la escuela secundaria, su opción es seguir la universidad. Las elecciones de seguir estudios superiores, de carrera y de destino (porque en sus ciudades o pueblos no hay universidad) implican un trabajo en el que se pone en juego lo subjetivo, lo social y lo cognoscente.

Hablamos de un proceso decisorio en el que el sujeto elige dentro un número finito de opciones, atribuye diferentes valores a las opciones, posee expectativas de futuro, imagina diferentes posibilidades en torno a la carrera, la universidad y, el lugar de destino. Las elecciones se van generando desde una decisión consciente, pero en el marco de lo que entendemos es una racionalidad limitada. Esto significa que el sujeto va definiendo en función de ciertas sujeciones, es decir, que se van generando valoraciones a partir de disposiciones construidas en proceso de 
interiorización de una exterioridad. Nos referimos a la noción de habitus (Bourdieu, 2007a), en tanto que las decisiones se hallan condicionadas por la trayectoria subjetiva y social de los agentes. Para este autor

El habitus es una capacidad infinita de engendrar con total libertad (controlada), unos productos-pensamientos, percepciones, expresiones, acciones- que siempre tienen como límite las condiciones histórica y socialmente situadas de su producción, la libertad condicionada y condicional que él asegura está tan alejada de una creación de novedad imprevisible como de una simple reproducción mecánica de las condiciones iniciales. (p. 90)

De este modo, las elecciones se encuentran condicionadas por la trayectoria individual y por la pertenencia social, siendo el habitus una estructura-estructurante que asegura la presencia activa (aunque no consciente) de las experiencias pasadas, que se actualiza en las decisiones y acciones presentes en función de las propensiones que se han generado en la práctica. En concordancia con lo anterior, queremos agregar que las disposiciones respecto del porvenir, la mirada a futuro, se han constituido en un universo particular de probables en relación, a su vez, de un estado determinado de probabilidades que el mundo social le asigna objetivamente (Bourdieu, 2007a). Cuando el joven elige carrera y destino para estudiar aparece ese juicio sobre lo probable en función de un principio de percepción selectiva constituido en su trayectoria vital.

Las elecciones realizadas por los sujetos en distintos dominios de la práctica, como puede ser estudiar, practicar deportes, ejecutar uno u otro instrumento, etc., se vinculan a las disposiciones estructuradas en el habitus, esquemas generados en función de las posiciones sociales (Bourdieu, 2007b). Dichas prácticas producen esquemas clasificatorios, principios de visión y división, gustos que operan como categorías al momento del proceso decisorio.

En resumen, nos parece sustantivo decir que, en las decisiones, aun cuando sean racionales, se juegan huellas disposicionales generadas a partir de las experiencias subjetivas y sociales de los sujetos, que se actualizan en contextos de acción diversos (Lahire, 2006). En dichas decisiones, intervienen entonces las experiencias socializadoras de los sujetos agentes, en las que participan con fuerza las instituciones familiares y escolares, en donde padres, docentes, pares y otros; son marco intersubjetivo activo en el proceso decisorio.

Otro concepto es la noción de interés vinculada a la de Illusio (Bourdieu y Waqcuant, 1995). Al contrario que la ataraxia, la Illusio refiere al hecho de estar involucrado, de estar atrapado en el juego y por el juego. Estar interesado quiere decir aceptar: lo que acontece en un juego social determinado, que sus apuestas son importantes y dignas de ser emprendidas antropológicamente, y que el interés es una arbitrariedad histórica, una construcción histórica. Por tanto, esto implica que existen tantos "intereses" como campos, que cada campo presupone y genera, de manera simultánea, una forma específica de interés inconmensurable con los intereses vigentes en otras partes.

Los sujetos no se encuentran "preocupados" por ciertos resultados futuros inscritos como posibilidades en el presente, sino en la medida en que su habitus los predispone y moviliza para perseguirlos. 
Cada campo define y activa una posición vinculada a intereses específicos según la trayectoria de cada sujeto, los cuales pueden interpretarse en el caso de los estudiantes entrevistados cuando se analizan las opciones, decisiones y expectativas con relación a la migración y a los estudios en la universidad

\section{Metodología}

El trabajo de investigación se ha planteado desde un enfoque cualitativo, que pretende conocer aspectos de las trayectorias de los/as jóvenes, desde el sexto año del secundario hasta los dos primeros años de arribo a la ciudad de Córdoba. Cobró valor este enfoque en virtud de poder estudiar procesos de transición como es el paso del nivel secundario a la universidad y el paso de vivir en familia a alejarse de la misma e integrarse a un nuevo lugar de acogida.

El trabajo se organizó en dos fases. La primera parte se realizó en el momento final de los estudios secundarios, entrevistando a aquellos/as estudiantes que tenían pensado movilizarse a la ciudad de Córdoba para iniciar sus estudios superiores. En esta fase, también se entrevistaron padres o madres de estos/as estudiantes. En la segunda fase, se estableció un nuevo contacto con aquellos/as jóvenes que efectivamente viajaron.

Para este artículo, solo nos centraremos en la información obtenida en la primera etapa. Se administraron entrevistas a los/as jóvenes durante el último semestre del sexto año a los fines de conocer cómo se van construyendo las elecciones de carrera y de ciudad de destino para estudiar en la universidad en jóvenes del interior de la provincia de Córdoba que están en sexto año de la escuela secundaria.

Se hicieron entrevistas en junio y noviembre a los/as jóvenes que estaban cursando el sexto de la escuela secundaria, a dos cohortes, una que finalizó en 2018 y otra en 2019, como así también a algunos de los padres o madres de estos estudiantes.

La elección de dichas instituciones se ha realizado contemplando la ubicación geográfica, para poder abordar escuelas de distintas regiones de la provincia. Por otro lado, se ponderó la factibilidad del trabajo de campo en dichos lugares en lo que fue la primera fase de la investigación. Los/as jóvenes cursaron el sexto año en escuelas ubicadas en cuatro regiones distintas: departamento San Justo, Punilla, San Alberto y Calamuchita.

Se realizaron doce entrevistas en la escuela ubicada en San Justo, seis entrevistas a estudiantes de la escuela ubicada en el Valle de Punilla, once en la tercera institución ubicada en San Alberto y doce en el Valle de Calamuchita.

Las entrevistas se han analizado desde una mirada interpretativa, lo que busca captar la lógica más profunda de lo social, de las experiencias estudiantiles, intentando sumergirse "en la particularidad de una realidad empírica, históricamente situada y fechada, pero para construirla como caso particular de lo posible" (Bourdieu, 2003, p.25). 


\section{Decisiones de las y los jóvenes, aspiraciones personales y familiares}

Si bien la decisión de continuidad de los estudios en la universidad puede ser identificada como una decisión de cada joven, la misma conlleva un proceso de elaboración con distintas aristas y en el que, aunque parezca que lo hace de modo individual, de ninguna manera lo logra en soledad. Es decir, hay discursos y referentes que de modo explícito o implícito afectan el proceso decisorio.

En un sentido semejante, Cleve (2014) sostiene que, si bien la acción de migrar es llevada adelante por los/as jóvenes de manera individual, puede constituir, a su vez, una estrategia familiar más amplia. Es decir, son las unidades familiares las que promueven la migración de sus miembros más jóvenes como estrategia económica y social.

En las entrevistas realizadas, se pueden identificar los modos en que las enunciaciones, tanto de los/as jóvenes como de sus padres, dan cuenta del entramado de sentidos que se juegan en este proceso. Sostenemos que, en las dudas, vaivenes, decisiones y cambios, participa la/el joven y aquellos referentes significativos que hacen de sostén intersubjetivo en esos momentos en los que se va definiendo "qué hacer" una vez finalizado el secundario. Ello, de algún modo, se ejemplifica en la siguiente enunciación:

"Siempre estuvieron preocupados a que sea redituable para mí. Que no gaste años de más, repitiendo materias. Por eso, me dieron la oportunidad de no trabajar a la hora de cursar. Van a bancar todo ellos y consideran que la educación es lo más importante que me pueden dejar, por eso quieren que estudie." (Carina, comunicación personal, Calamuchita, 2019).

Por otro lado, la mayoría de los padres mantiene que la elección académica realizada es una responsabilidad de los/as hijos, la que, en buena medida, condicionará su vida futura. Como consecuencia de esa interpretación, algunos aclaran que no imponen nada como obligatorio ni les presionan en ningún sentido:

"Y nosotros...es la primera que se va a Córdoba, entonces, también muchas expectativas, es

la más grande de sus primos, entonces, también, como que los abuelos, los tíos, los primos están todos... ahí... viendo qué va a hacer ella. Pero no... Siempre acompañándola y ayudándola a tomar las decisiones, nada impuesto. Ella elige." (Juana-madre-, comunicación personal, San Justo, 2018).

Los/as estudiantes también expresan que han tenido márgenes de libertad y de apoyo para cada momento en que han definido posibilidades tanto en lo vinculado al desplazamiento como a los estudios:

"Sí me apoyan. Siempre me apoyaron en tema de carrera, nunca trataron de inculcarme alguna carrera en especial así que..." (Ludmila, comunicación personal, Calamuchita, 2018).

Para algunos/as jóvenes, las decisiones se vivencian como libre de condicionamientos, al menos explícitos. Sin embargo, en las elecciones se entrelazan aspectos que pueden haber jugado sin que haya plena conciencia de estos. No hay, como dice el joven citado anteriormente, una inculcación evidente. Para las figuras parentales, pareciera primar la necesidad de una opción libre que pudiera responder a los deseos de los hijos. Situación que, como se lee en la siguiente 
expresión, requiere del reconocimiento, en palabras y gestos, que oficia de sostén de dichos padres. Hay libertad sujetada a un imprescindible registro de apoyo:

"Están contentos con la decisión, siempre me dieron la posibilidad de poder elegir y la libertad también. Que haga lo que me gustara. Lo fundamental es tener el apoyo de ellos" (Ariadna, comunicación personal, Calamuchita,2018).

Persiste, en algunas familias, la histórica apuesta a la educación como fuente de movilidad social. Apuesta mayor y más incierta en el caso de padres y madres que no han tenido estudios universitarios superiores y que desean que sus hijos/as sí los tengan. Dos experiencias diferencian a estos grupos de familias de aquellas en que hay experiencias migratorias ligadas a estudiar en la universidad.

"Sí por supuesto, uno tiene la mejor expectativa. Lo mejor es que el elija bien. Que le guste, que se sienta cómodo. Uno quiere que le vaya bien. Una cosa que no pudimos hacer nosotros. Nosotros le vamos a brindar eso." (Mónica -madre-, comunicación personal, San Justo, 2018).

Expectativas, esfuerzo, investimento libidinal por iniciar un juego social (desplazamiento y universidad) del que no conocen mucho. Algunos de estas/os jóvenes corresponden a aquellos/as que se los denomina primera generación familiar en la universidad.

"Y mi familia está de acuerdo con eso, porque sería como una de las primeras que tendría un título de mi familia, y les gustó mucho la idea de Arquitectura, más para esta zona, que se hacen muchas construcciones, y todo eso..." (Victoria, comunicación personal, San Alberto, 2019).

Por otro lado, está el grupo de jóvenes en donde la universidad es un itinerario previsible, dado que, desde la infancia, se habla de las propias experiencias de los padres, sus estudios y desempeños laborales vinculados a ese recorrido.

"Va a estudiar Medicina. Su papá es médico, yo soy fonoaudióloga y bueno, ella desde el primario que está decidida a estudiar medicina, por eso también se vino a este colegio (...), yo le sugerí que escuchara las tres charlas por las dudas que hubiera algún cambio y bueno, se mantiene firme con esa decisión." (Madre-Adriana, comunicación personal, San Justo, 2019).

Las expectativas hacen referencia a un compromiso con alcanzar determinado nivel educativo; es decir, suponen un plan sobre cómo lograr esa meta. Se trata de un escenario en el que se evalúan las posibilidades que tiene un sujeto en particular de alcanzar determinado nivel educativo, considerando tanto la información disponible en el momento actual como sus preferencias. Es decir, las expectativas son susceptibles a influencias externas, pues toman en consideración los recursos económicos disponibles, el soporte familiar existente y otros factores que pueden afectar las oportunidades educativas (Guerrero, 2014). 


\section{Pensar-se en la nueva ciudad y en la universidad}

Pensar-se en la ciudad en el último año de la escuela secundaria supone, en algunos/as estudiantes, ir proyectando su mudanza a un lugar desconocido o un traslado a una ciudad que solo ha sido visitada en algunas ocasiones. Hablamos así de transiciones que se van concretando en itinerarios vitales que configuran los trayectos (Rascovan, 2014).

Es un dinamismo que supone un cambio de ritmo o rutina en la jornada habitual, va de la mano con las expectativas e inicio de algo desconocido. Citamos algunas expresiones, como referencias propias de los/as jóvenes respecto de expectativas, desafíos y pérdidas que, piensan, deberán afrontar:

"Ansiedad, tenía ganas de terminar el colegio y ya estar ahí" (Javier, comunicación personal, Calamuchita, 2019).

"Tengo un montón de ilusiones y también muchas contras! Me da mucho miedo a no ingresar. No le tengo miedo a vivir sola o a manejarme, a eso me acostumbro y además me gusta estar sola en un ambiente. Pero le tengo mucho miedo al ingreso. Tengo mucho miedo." (Marco, comunicación personal, San Alberto, 2019)

Los/as estudiantes han estado acostumbrados a una escolaridad obligatoria bastante previsible en la que se encontraban acompañados por los pares, constituyendo un grupo sostenido en el tiempo. Ahora, se ven inmersos en un momento de decisión personal, que cada uno transita en función de rasgos distintos y de experiencias particulares. El desafío es poder decidir qué estudiar. Luego, dónde. Se abre una nueva etapa:

"Bien, eh, me duele por un lado porque voy a extrañar el grupo, todo, pero siento también que ya es necesario empezar con algo nuevo. Empezar con otra etapa. Si me decís que el año que viene tengo que volver acá es un bajón, ya siento que se tiene que cerrar la etapa, que necesito algo nuevo." (Erika, comunicación personal, Calamuchita, 2019).

En esta proyección de pensar-se en la ciudad, aparecen algunas construcciones de representaciones referidas a sí mismo y al mundo de las ocupaciones y profesiones que están íntimamente relacionadas con la propia biografía escolar, de manera tal que las variadas experiencias desarrolladas a lo largo de su trayecto educativo son condicionantes en el proceso de construcción futuro (Rascovan, 2014).

La próxima independencia, nuevas obligaciones, cambios en el ritmo de vida, logro de autonomía y nuevo lugar donde comenzar a vivir se expresan cuando comparan entre lo vivido y lo proyectado, lo futuro a venir:

"Voy a tener más obligaciones. Tener que ser más responsable. Voy a tener que manejarme solo" (Esteban, comunicación personal, San Alberto, 2019);

"Los primeros meses me los imagino mal, porque es una vida totalmente distinta. No totalmente distinta a ser independiente ya que estoy acostumbrada, sino a que dejas de ser una niña. Yo acá, en el colegio, me conoce todo el mundo, soy S, soy la bailarina, la ex presidenta del centro de estudiantes. Cumplí muchas funciones, los profes me aman, todos me conocen y como que, en la universidad, somos un número y como que eso también me 
cuesta un poco. No voy a decir: me voy al baño del brazo de mi amiga. No, es algo totalmente independiente. A eso le tengo miedo." (Soledad, comunicación personal, San Alberto, 2019); "En Córdoba, voy a estar más perdida porque no conozco tanto el tema... también de los colectivos, todo, me tengo que manejar así o caminando" (Micaela, comunicación personal, San Justo, 2019).

Especie de adaptabilidad proyectada, que se relaciona con el deseo del estudiante. En este proceso, aparecen los miedos por temor a no poder lograrlo.

El miedo de no poder responderá a estos nuevos intereses que serán sostenidos desde la Illusio del/la estudiante en su proceso nuevo de construcción simbólica tanto de relaciones con los otros como de él mismo en su contexto. Lo que sabe del nuevo espacio urbano, lo que sabe de la universidad, lo que sabe de vivir lejos de la familia. Representaciones que han ido ofreciendo otros/as sujetos desde experiencias semejantes previas que se suman las imágenes e imaginación de los/as jóvenes prontos a partir para estudiar:

"No adaptarme en la ciudad. Al no estar acostumbrada, tengo miedo de no adaptarme y querer volverme o no adaptarme a la rutina, a la cantidad de horas de estudio..." (Micaela, comunicación personal, San Justo, 2019);

"Es muy tranquila, que eso también, por ahí siento que acá, si tengo que hacer algo en el centro, me voy, no sé, en bici o caminando y allá, en Córdoba, voy a estar más acelerada, digamos, o no sé, estar todo el tiempo yendo y viniendo, esa es la idea que me hago, como que en el pueblo es más tranquilo". (Agustina, comunicación personal, San Justo, 2019);

"Bueno, a veces lo pensé, pero no, no me hago mucho la idea... pienso, me gustaría tener algunas amigas que vivan cerca del departamento, hacerme amigos nuevos allá para estudiar también, y... y bueno, no sé (risas), adaptarme" (Mariana, comunicación personal, San Justo, 2019).

La universidad es un escenario social, cultural, político, ético-estético y cognitivo donde se comparten experiencias, teorías y sensibilidades que pretenden ayudar a mantener, construir y desarrollar al sujeto, la sociedad y la cultura (Murcia, 2006).

Pensar-se en esa institución como nueva etapa la cual supone abrir un tiempo de socialización sobre nuevas reglas y prácticas, que se deberá aprender. Es una etapa donde se establecen nuevos vínculos, hay una nueva organización para poder vivir en una ciudad. Desde la búsqueda de departamentos, residencias, ver las distancias y medios de transportes para poder llegar a la universidad. También consideran importante establecer algún contacto con familiares, personas conocidas o cercanas al círculo familiar para poder acudir frente a alguna situación particular en la nueva ciudad de acogida.

Entre los/as estudiantes entrevistados, encontramos discursos en los cuales se piensan en la universidad como un caminando, un camino que implica crecimiento e independencia. La escuela secundaria les da un parámetro de comparación con relación al nuevo desafío que deben emprender. Encontramos expresiones tales como: 
"Estoy acostumbrada que es todo más relajado en el colegio y allá es todo más independiente, autónomo, tenés que hacer la cosas por tu cuenta, siento que voy a estar muy estresada. Son varias materias, temas que tienen que ver, pero al mismo tiempo no tanto. También creo que voy a ser muy feliz porque es lo que me gusta". (Laura, comunicación personal, Calamuchita, 2019).

Cuando se proyectan en el mundo universitario, lo asocian fundamentalmente al desafío intelectual y a las exigencias académicas, estableciendo diferencias con la escuela secundaria donde el peso, en sexto año, estuvo puesto más en lo social. A su vez, experimentan inquietudes como: ¿qué quiero ser?, ¿voy a poder?, y, para ello, ¿qué debo o deberé realizar o hacer?

"Se siente raro porque estás acostumbrado a tener tu entorno en el colegio. Llegas a las 7:50 horas y ves a tus compañeros y te vas, es como una rutina que se hace porque son seis años que estás con las mismas personas en la misma rutina y tener que cambiarlo todo es como un shock bastante fuerte para todos. Estamos como todos tristes porque estás terminando una etapa importante de tu vida y quizás no veas nunca más a alguien, es como re fuerte para todos." (Laura, comunicación personal, Calamuchita, 2019).

En síntesis, podemos decir que las ideas, ilusiones, creencias y proyectos al finalizar la escuela secundaria se vinculan a expectativas, posibilidades anticipadas, percepciones de obstáculos, entre otros, lo que conforma un conjunto de representaciones elaboradas a lo largo de su vida social. El paso por las instituciones escolares y el papel de la institución familiar no son ajenos a la construcción de las representaciones sobre el futuro. Serán las decisiones individuales, sostenidos por la vivencia grupal, en conjunto a sus motivaciones e intereses, lo que posibilitará en los estudiantes la concreción de este gran paso.

\section{Anticipando la migración. Condiciones materiales y movilizaciones subjetivas}

La mirada referida al futuro implica aquello que se espera como posible o aquello que puede ser proyectable sin que necesariamente se tenga certeza de alcanzarlo totalmente. Estas proyecciones no se dan en el vacío, los/as estudiantes no están aislados del contexto en el cual desarrollan sus expectativas. La selección subjetiva del camino a recorrer tendrá mayores o menores posibilidades de ser llevadas a cabo en función de las restricciones que les imponga el contexto objetivo en el cual viven (Corica, 2012).

Las entrevistas analizadas nos permiten interpretar de qué modo las condiciones socioeconómicas, educativas y culturales de las familias condicionan, posibilitando u obstaculizando la iniciación del proyecto educativo universitario.

Retomando a Bourdieu (2010), el autor menciona que los agentes se distribuyen en el espacio social según el volumen de capital. De este modo, se constituye en red de posiciones y relaciones. Cada posición en el campo se explica en relación con otras posiciones en virtud de la distribución desigual de los recursos, es decir, de los capitales. En esta ocasión, vamos a hacer hincapié en el capital económico y cultural. 
Los factores de orden económico condicionan la disponibilidad familiar para asumir los diversos gastos que se derivan del traslado: alojamiento, alimentación, material de estudio, acceso a servicios y movilidad. Algunos/as jóvenes tienen la posibilidad de que sus padres carguen con los gastos y mantenimiento de los años de estudios universitarios. La distinción por lo económico es un dato destacable: los que tienen más oportunidades de progresar son los/as jóvenes que están en mejor posición económica (Corica, 2012):

"Tengo pensado mudarme con mi mejor amiga en un departamento para que los gastos sean más Ilevaderos" (Laura, comunicación personal, Calamuchita, 2019);

"Estoy viendo un departamento que me dijeron que está entre medio del jockey de Córdoba, el club de rugby ese y la nacional. Me dijeron que es bastante lejos, pero no sé, e igual me da lo mismo dónde queda porque lo que quiero es estudiar sí o sí. Tengo la posibilidad que el padre de un amigo me puede facilitar un trabajo y todo." (Emiliano, comunicación personal, Punilla, 2019).

Por otra parte, los factores culturales y las trayectorias educativas parentales inciden, en tanto referentes identificatorios, en la valoración y el apoyo que se asigne a los estudios universitarios como proyecto de vida, por lo tanto, se configura como un contexto posibilitador de las elecciones y aspiraciones. Las opciones realizadas por los/as estudiantes se vinculan a las disposiciones estructuradas en el habitus, esquemas generados en función de las posiciones sociales (Bourdieu, 2003). La estructura del hogar incide significativamente las probabilidades de tener expectativas de educación superior (Guerrero, 2014):

"Me alegra por la etapa que va a transitar, no me deprime...-mi estancia en Córdoba fue muy buena...yo viví cinco años, la pasé re bien, nunca tuve contratiempos, pude ir y venir cuando quise, los fines de semana que podía venir, no hay por ahora limitantes económicos que resolver, entonces, creo que no le va a faltar nada, que todo lo que ella haga va a estar bien, que la voy a tener que limitar un poco, eso sí. Pero no creo que le vaya mal, que no lo pueda resolver. Nunca le exigí nada. Pero creo que, en la forma que tenemos de convivencia y demás, ella sabe lo que esperamos de ella, sin que se lo diga, y tiene capacidad. No es una chica que al segundo día se vaya a largar a llorar...-me va a llamar... y voy a estar si me necesita. Pero que creo que va a estar bien. Que lo va a transitar bien como lo transité yo y mi marido, que estuvimos cinco años en Córdoba." (Carina-madre, comunicación personal, San Justo, 2018).

Las expectativas de los padres y las madres se traducen en la percepción que tienen los/as hijos/as sobre sus propias habilidades y, posteriormente, en sus expectativas educativas; así, altas expectativas educativas por parte de los padres se convierten en una percepción positiva de los hijos respecto a sus habilidades y, consecuentemente, en altas expectativas educativas (Guerrero, 2014).

Otro aspecto altamente significativo en el proceso migratorio que inician estos/as jóvenes es de orden subjetivo, en cuanto a la capacidad de desprendimiento y reconocimiento, por parte de los/as progenitores y del mismo/a estudiante, del proceso de autonomía y responsabilidad que 
debe asumir este último para materializar adaptativamente los cambios que representa esta movilidad en su vida cotidiana. Algunos/as jóvenes transitan estas experiencias como procesos dolorosos que generan sufrimiento, desarraigo y dificultad para tramitar el duelo por las pérdidas inevitables (culturales, lazos sociales, afectivos, etc.) Otros y otras estudiantes transitan dicha experiencia con mucha expectativa e ilusión.

"Em... no sé, es como si me decís ahora tengo ganas de irme un montón, qué se yo... pero todos los que fueron allá me dicen: "el primer año vas a extrañar, te vas a querer volver". No sé, yo tengo ganas de irme para allá, para ver cómo es, todo." (Lucila, comunicación personal, San Justo, 2018).

Como mencionamos en un inicio, la decisión de comenzar los estudios en la universidad conlleva un proceso de elaboración con distintas aristas y en el que, aunque parezca que lo hace de modo individual, los procesos decisorios se van dando en una trama intersubjetiva en que se conjugan aspectos psicosociales que condicionan dichas decisiones.

Los/as jóvenes se valen de otras experiencias ajenas para ir imaginando la vida en la nueva ciudad y los posibles desafíos en la universidad. Algunos cuentan con relatos de referentes significativos más próximos como padres, madres, hermanos y hermanas que ya han transitado ese proceso y otros pertenecen a la primera generación que proyecta esta posibilidad migratoria con el objetivo de iniciar sus estudios superiores. Las trayectorias anteriores y los itinerarios que se trazan como posibilidad se vinculan con condiciones objetivas, como así también con aspectos subjetivos de cada joven en singular.

\section{Al cierre}

En el mes de septiembre del corriente año, el Concejo Deliberante declaró a Córdoba como Ciudad Amigable del Estudiante Universitario. Algunos de los argumentos dados en dicha sesión se vincularon con la tradición universitaria, por la cual se conoce esta ciudad como la Docta. Alberga, en el centro de la ciudad y en barrios aledaños, al menos unos 100.000 estudiantes provenientes de distintos puntos del país. Este hecho, de algún modo, ilustra lo que representa para los/as jóvenes migrar hacia Córdoba para estudiar.

El proceso decisorio toma distintas formas en cada sujeto, pero participan de modo activo las familias, las instituciones educativas y las y los pares. Dos decisiones trascendentales se barajan para estas/os jóvenes: elegir qué estudiar y elegir dónde. Cuentan con algunas certezas, pero más pesan las incertidumbres que abren desafíos, tiñendo los discursos de ansiedad, temores y deseo de concreción de los planes que vienen pensando. Los referentes adultos acompañan este proceso y las condiciones objetivas de posibilidad difieren de una familia a la otra. El estudio longitudinal permite acompañar a estas/os jóvenes por dos años más con el objetivo de conocer los cambios y las vicisitudes que se dan tanto en lo que respecta al proceso migratorio como al ingreso y continuidad de los estudios superiores.

La investigación que venimos realizando procura dar visibilidad a los procesos migratorios internos que, desde las familias y escuelas del interior, muchas veces se naturalizan como lo dado 
necesariamente. Identificar aspectos singulares de este proceso podría aportar elementos para producir, desde las Instituciones de los Niveles Secundario y Superior, formas articuladas de abordaje, creando o utilizando espacios institucionales ya existentes para trabajar anticipadamente con aquellos/as estudiantes que tendrán que mudarse hacia Córdoba Capital para iniciar estudios superiores.

La investigación tiene como perspectiva dar algunas respuestas a problemas que surgen en los propios procesos de la vida universitaria, vinculados a aspectos que se desconocen o que han sido poco objetivados en torno a lo que los/as estudiantes efectivamente vivencian en situaciones de migración, dada la distancia entre los mundos de la vida de los lugares de origen y las formas de acción propias de la gran ciudad. Desde las instituciones educativas, se pueden pensar intervenciones que podrían beneficiar al sostenimiento de la carrera, proveyendo espacios de acompañamiento que ofrezcan contención psicosocial, aspecto indispensable para la construcción de conocimiento. Ello también es posible si hay sostén intersubjetivo fundamentalmente durante el primer período de la migración en el que se producen los mayores ajustes de adaptación al nuevo espacio.

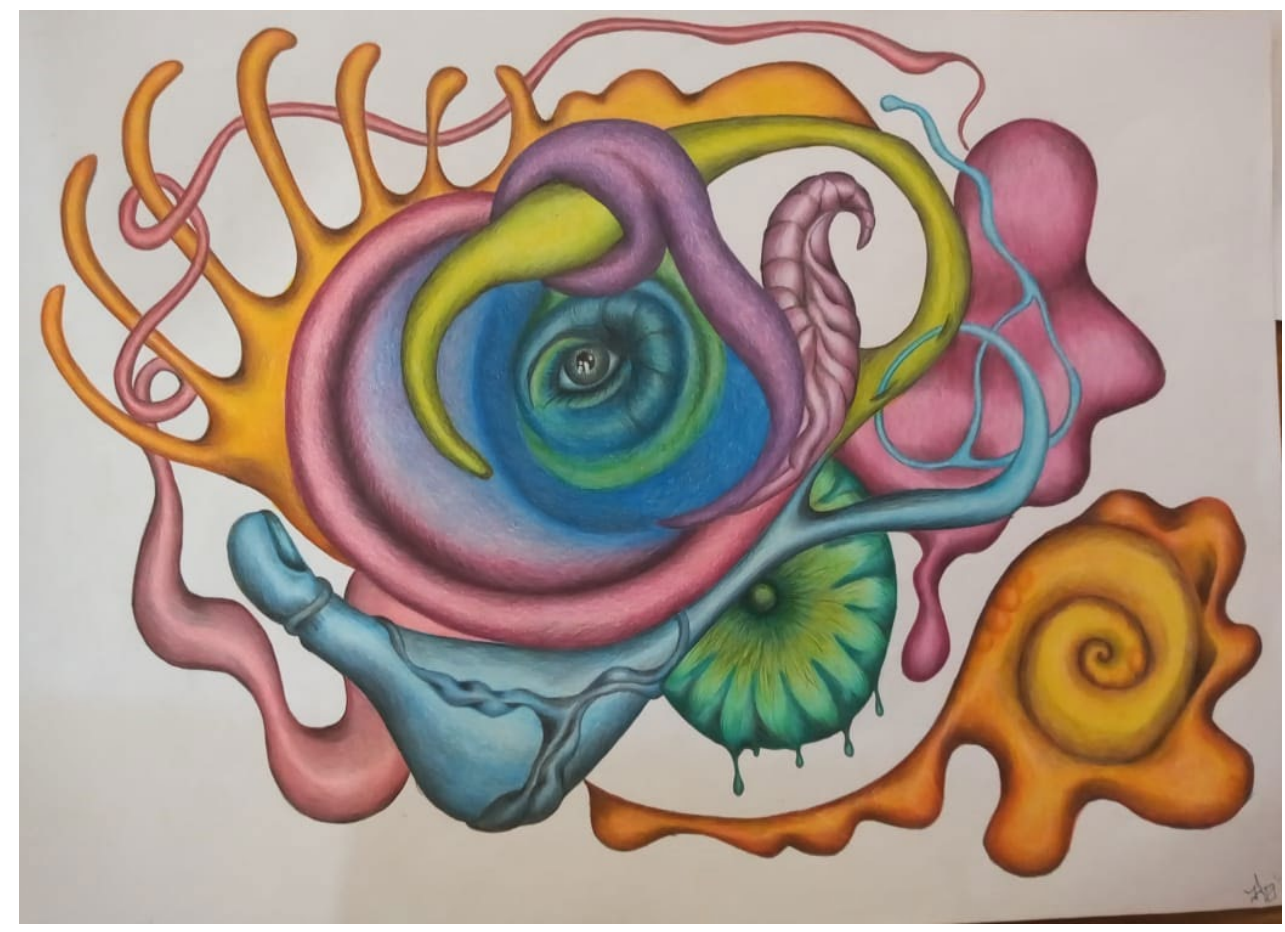

Sin título, lápices sobre hojas. Romina Solange Finks

\section{Bibliografía}

Bourdieu, P. y Waqcuant, L. (1995). Respuestas: por una antropología reflexiva. Ed. Grijalbo.

Bourdieu, P. (2007a). El sentido práctico. Siglo XXI.

Bourdieu, P. (2007b). Razones prácticas. Sobre las teorías de la acción. Anagrama. 
Bourdieu, P. (2010). Capital Cultural, escuela y espacio social.

Camarena, R. (2000). Los jóvenes y la educación: Situación actual y cambios intergeneracionales. Papeles de población, 6(26), 25-41 http://www.scielo.org.mx/scielo.php?script=sci_arttext\&pid=S1405$74252000000400003 \&|n g=e s \& t| n g=e s$.

Cleve, A. (2014). Me voy a estudiar a La Plata: sentidos y prácticas diversas en jóvenes que migran de ciudad para comenzar la Universidad. XI Congreso Argentino de Antropología Social. Facultad de Humanidades y Artes, Rosario.

Cleve, A. (2016). El lugar de las familias en las migraciones internas de estudiantes hacia La Plata, Argentina: apoyo económico, acuerdos y construcción de redes. Odisea. Revista de Estudios Migratorios, 3, 5377.https://publicaciones.sociales.uba.ar/index.php/odisea/article/view/1945

Corica, A. (2012). Las expectativas sobre el futuro educativo y laboral de jóvenes de la escuela secundaria: entre lo posible y lo deseable. Revista Última Década, 20(36), 7195.https://scielo.conicyt.cl/scielo.php?script=sci_arttext\&pid=S0718-22362012000100004

D'Angelo, O. (2001). Proyecto de vida como categoría básica de interpretación de la identidad individual y social. Revista Cubana de Psicología, 17(3), 270-275. http://biblioteca.clacso.edu.ar/Cuba/cips/20120827125359/angelo8.pdf

Erikson, E. (1968). Identidad, Juventud y Crisis.Editorial Paidós.

Gómez, S., Etchegorry, M. y Caón, C. (2017). Despliegue de acciones frente a las experiencias transitadas al inicio de la vida universitaria: la tensión entre lo conocido y lo novedoso. Revista Pilquen, 14(2), 15-29. http://revele.uncoma.edu.ar/htdoc/revele/index.php/psico/article/viewFile/1796/1833

Gómez, S., Etchegorry, M., Avaca, F. y Caón, C. (2016) Trayectos escolares previos y vida universitaria. Revista Praxis Educativa, 20(3), 38-46 https://cerac.unlpam.edu.ar/index.php/praxis/article/view/1359/1431

Gómez, S. (2019). Experiencias migratorias de estudiantes universitarios. Revista Praxis educativa, 23(1), 1-13. http://www.biblioteca.unlpam.edu.ar/pubpdf/praxis/v23n1a08gomez.pdf

Guerrero, G. (2014). Yo sé que va a ir más allá, va a continuar estudiando: expectativas educativas de estudiantes, padres y docentes en zonas urbanas y rurales del Perú. GRADE

Lahire, B. (2006). El espíritu sociológico. Manantial.

Mercado, R. (2014). Estudiantes migrantes y políticas de inclusión en la universidad pública. XI Congreso Argentino de Antropología Social. Facultad de Humanidades y Artes, Rosario.

Murcia Peña, N. (2009). Vida universitaria e imaginarios: posibilidad en definición de políticas sobre educación superior. Revista Latinoamericana de Ciencias Sociales, Niñez y Juventud, (11), 235-266. https://www.redalyc.org/articulo.oa?id=773/77307110

Pucci, R. (2018). Factores que inciden en el proceso de toma de decisión vocacional en jóvenes del interior que migran para continuar estudios superiores (tesis de Licenciatura). Universidad Católica Argentina, Buenos Aires.

Rascovan, S., Levy, D. y Korinfeld, D. (2014). Entre Adolescentes y Adultos en la Escuela. Puntuaciones de época. Paidós.

Rascovan, S. (2015). Los jóvenes y el futuro. Noveduc. 